\title{
The issue of how predict survival of patients affected by locally advanced pancreatic cancer
}

\author{
Benedetto Ielpo \\ HPB Unit, General Surgery, University Hospital of Leon, Calle Altos de Nava s/n León, Spain \\ Correspondence to: Benedetto Ielpo, MD, PhD, FACS. HPB Unit, General Surgery, University Hospital of Leon, Calle Altos de Nava s/n León, Spain. \\ Email: ielpo.b@gmail.com. \\ Provenance: This is an invited Editorial commissioned by Section Editor Long Pan (Zhejiang University, Hangzhou, China). \\ Comment on: Choi SH, Park SW, Seong J. A nomogram for predicting survival of patients with locally advanced pancreatic cancer treated with \\ chemoradiotherapy. Radiother Oncol 2018;129:340-6.
}

Submitted Nov 28, 2018. Accepted for publication Dec 14, 2018.

doi: $10.21037 / \mathrm{atm} .2018 .12 .41$

View this article at: http://dx.doi.org/10.21037/atm.2018.12.41

I've read with great interest the paper of Choi et al. (1) which may be of great interest among the oncological and surgical community.

It is well known that pancreatic adenocarcinoma (PA) is still considered a malignancy with an extremely poor prognosis (2). Only $15-20 \%$ of patients at the initial diagnosis are suitable for pancreatectomy as the most present with metastases or are locally advanced (3).

The neoadjuvant benefit for resectable patients remains a highly controversial issue and only in the last years, with the development of new drugs and strategies, few studies address it with some slight improved overall survival (OS) (4-6).

On the other hand, neoadjuvant treatment for locally advanced PA, which is the topic of this paper, is currently the standard of care.

Talking about localized and resectable PA may rise some confusion as several PA staging exist $(2,7)$. However, all these staging systems considers some common points as follow.

Localized PA can be classified into the three following categories based on vascular involvement by the tumor assessed on cross-sectional imaging: potentially resectable (PR: absence of vascular involvement), borderline resectable (BR: minor/moderate vascular involvement), and locally advanced (LA: unreconstructable venous occlusion or significant arterial involvement).

However, the study we are commenting on and which authors are aware, included in their analysis both BR and LA tumors, which may lead to some confusion when results are discussed. It would have been very interesting to have the results of the two groups of PA in order to better understand the analysis of this study. If it is clear that for LA PA the neoadjuvant treatment is paramount. On the other hand, for BR PA the benefits are still under investigation and in my opinion, still most of the centers did not give preoperative chemotherapy. In my previous study (5), comparing neoadjuvancy vs. upfront surgery in $\mathrm{PA}, \mathrm{OS}$ and disease-free survival were higher in the BR PA group of patients receiving preoperative treatment. Furthermore, almost $40 \%$ of patients did not underwent to adjuvant treatment because of postoperative complications. In this latest group, only those who received preoperative treatment, by controlling underlying disease, had the chance of higher OS and disease-free survival.

For patients affected by LA PA, chemotherapy was considered almost palliative with a minimum chance of being resectable. However, more recently, a better understanding of the molecular biology of PA has allowed the development of novel chemotherapeutic agents and combinations such as FOLFIRINOX and gemcitabine/nabpaclitaxel based treatment with or without combination with concomitant radiotherapy $(5,6)$. These new protocols are showing an improved radiological response with increased OS in metastasic PA and increased resection rate in LA PA compared with the past $(5,6,8-10)$. For this reason, with the aim of predicting the response of neoadjuvant treatment for LA PA, the topic of this paper is of great interest.

In the last years, it has been showed as RECIST criteria 
is unable to predict alone the chemotherapy response, and alternative response criteria have been proposed in PA like CA 19.9 decrease, standardized uptake value (SUV) uptake decrease at FDG-PET scan (11-13). However, up to know, none study has ever analyzed the predictive accuracy combination of these values, such as in the herein paper by Choi et al. (1).

Including 426 patients affected by LA PA, one of the strengths of this study is the high population number. All previous studies include less than 50 patients with LA PA cases being mostly multicentric, therefore, with some missing data and biases.

Prognostic value of SUV uptake has been already studied, and its accuracy is still under investigation. Some studies proved the prognostic value in some malignancies such as in PA $(12,14)$. However, the SUV decline rate, which measure the decrease of the SUV value after completing the neoadjuvancy, has not been well investigated yet.

The present study, shows its value on predicting response only when initial SUV is minor of 3.5. However, in my opinion, the time when FDG-PET scan is performed after finishing the treatment is paramount for prognostic purpose. It is not the same if the scan is performed in an early period of time (1-2 months after the end of the treatment) or later. I would expect that neoadjuvant effect after completing treatment may follow a curve type fashion being higher in a specific period time which is pending to be assessed yet. Recently, in rectal cancer, it has been showing that resection performed 4-6 months from the end of neoadjuvant treatment is associated with higher diseasefree survival (15). Therefore, in my opinion, the higher effect of neoadjuvancy in LA PA as well as in most of the malignancies, might be detected better after 1 month from the treatment.

The study of Choi et al. (1), did not define this period in their analysis (being performed within 12 months), that on the contrary would have given valuable information about when the effect of neoadjuvant treatment is higher and therefore when it is better measure the SUV value.

The role of radiation therapy in the management of LA PA remains controversial and results are divergent. The last available trial (LAP07) reported that, among patients with LA PA with disease controlled after 4 months of gemcitabine-based induction chemotherapy, there was no significant difference in OS with chemoradiotherapy compared with chemotherapy alone (16). Radiotherapy in PA have been well studied in previous series where more disadvantages than benefit have been reported due to its toxicity. However, in the last years, the 3- to 5-fraction lowdose stereotactic radiation (SBRT) adapted to respiratory motion, have likewise had a minimal impact on toxicity, showing an improved response on cancer. Maybe, also the fractions as well as doses might predict survival in LA PA.

Currently, radiation therapy delivered in PA has a median dose of $54 \mathrm{~Gy}$, which is consistent with the median 53.2 Gy detected in the present paper (1). However, a dose $>61$ Gy have also been found by the authors to be related with increased OS. On the basis that a higher radiation dose is deserved to larger and more advanced tumors, I would have expected that higher doses are related to worst outcomes. According to their experience, the authors should discuss in which cases of LA PA the radiation doses is increased from the standard level. Furthermore, the number of fractioning is also important. More fractions may allow higher doses to be given. This point should be analyzed in future researches.

The total number of chemotherapy cycles have been investigated too, and few data are available in the literature concerning its relation with OS (17). However, the number of chemotherapy cycles have not been investigated in this study and this number, as well, can be included in the nanogram for predicting survival.

In conclusion, this is a valuable paper that may permit to better stratify the prognosis of patients affected by initially unresectable PA. However, further studies are required to back up this result.

\section{Acknowledgements}

None.

\section{Footnote}

Conflicts of Interest: The author has no conflicts of interest to declare.

\section{References}

1. Choi SH, Park SW, Seong J. A nomogram for predicting survival of patients with locally advanced pancreatic cancer treated with chemoradiotherapy. Radiother Oncol 2018;129:340-6.

2. Tempero MA, Malafa MP, Al-Hawary M, et al. Pancreatic Adenocarcinoma, Version 2.2017, NCCN Clinical Practice Guidelines in Oncology. J Natl Compr Canc Netw 2017;15:1028-61. 
3. Winter JM, Brennan MF, Tang LH, et al. Survival after resection of pancreatic adenocarcinoma: results from a single institution over three decades. Ann Surg Oncol 2012;19:169-75.

4. Cooper AB, Parmar AD, Riall TS, et al. Does the use of neoadjuvant therapy for pancreatic adenocarcinoma increase postoperative morbidity and mortality rates? J Gastrointest Surg 2015;19:80-6.

5. Ielpo B, Caruso R, Duran H, et al. A comparative study of neoadjuvant treatment with gemcitabine plus nab-paclitaxel versus surgery first for pancreatic adenocarcinoma. Surg Oncol 2017;26:402-10.

6. Ielpo B, Duran H, Diaz E, et al. Preoperative treatment with gemcitabine plus nab-paclitaxel is a safe and effective chemotherapy for pancreatic adenocarcinoma. Eur J Surg Oncol 2016;42:1394-400.

7. Kleeff J, Korc M, Apte M, et al. Pancreatic cancer. Nat Rev Dis Primers 2016;2:16022.

8. Von Hoff DD, Ramanathan RK, Borad MJ, et al. Gemcitabine plus nab-paclitaxel is an active regimen in patients with advanced pancreatic cancer: a phase I/II trial. J Clin Oncol 2011;29:4548-54.

9. Hidalgo M, Plaza C, Illei P, et al. O-0004 SPARC analysis in the phase III MPACT trial of nab-paclitaxel (NAB-P) plus gemcitabine (GEM) vs GEM alone for patients with metastatic pancreatic cancer (PC). Ann Oncol 2014;25:ii105-17.

10. Conroy T, Desseigne F, Ychou M et al. FOLFIRINOX versus gemcitabine for metastatic pancreatic cancer. $\mathrm{N}$ Engl J Med 2011;364:1817-25.

11. Lim I, Noh WC, Park J, et al. The combination of FDG

Cite this article as: Ielpo B. The issue of how predict survival of patients affected by locally advanced pancreatic cancer. Ann Transl Med 2018;6(Suppl 2):S128. doi: 10.21037/atm.2018.12.41
PET and dynamic contrast-enhanced MRI improves the prediction of disease-free survival in patients with advanced breast cancer after the first cycle of neoadjuvant chemotherapy. Eur J Nucl Med Mol Imaging 2014;41:1852-60.

12. Choi HJ, Lee JW, Kang B, et al. Prognostic significance of volume-based FDG PET/CT parameters in patients with locally advanced pancreatic cancer treated with chemoradiation therapy. Yonsei Med J 2014;5 5:1498-506.

13. Koom WS, Seong J, Kim YB, et al. CA 19-9 as a predictor for response and survival in advanced pancreatic cancer patients treated with chemoradiotherapy. Int J Radiat Oncol Biol Phys 2009;73:1148-54.

14. Shankar LK, Hoffman JM, Bacharach S, et al. Consensus recommendations for the use of $18 \mathrm{~F}-\mathrm{FDG}$ PET as an indicator of therapeutic response in patients in National Cancer Institute Trials. J Nucl Med 2006;47:1059-66.

15. Imyanitov EN, Yanus GA. Neoadjuvant therapy: theoretical, biological and medical consideration. Chin Clin Oncol 2018;7:55.

16. Hammel P, Huguet F, van Laethem JL, et al. Effect of chemoradiotherapy vs chemotherapy on survival in patients with locally advanced pancreatic cancer controlled after 4 months of gemcitabine with or without erlotinib: the LAP07 randomized clinical trial. JAMA 2016;315:1844-53.

17. Pietrasz D, Turrini O, Vendrely V, et al. How Does Chemoradiotherapy Following Induction FOLFIRINOX Improve the Results in Resected Borderline or Locally Advanced Pancreatic Adenocarcinoma? An AGEOFRENCH Multicentric Cohort. Ann Surg Oncol 2018. [Epub ahead of print]. 